\title{
Virtual coronary angioscopy using multislice computed tomography
}

\author{
S Schroeder, A F Kopp, B Ohnesorge, H Loke-Gie, A Kuettner, A Baumbach, C Herdeg, \\ C D Claussen, K R Karsch
}

See end of article for authors' affiliations

Correspondence to:

Dr Stephen Schroeder,

Medical Clinic, Division of

Cardiology, University of

Tuebingen,

Otfried-Mueller-Strasse 10,

D-72076 Tuebingen,

Germany;

stephen.schroeder@

med.uni-tuebingen.de

Accepted 26 September

2001
Background: With faster image acquisition times and thinner slice widths, multislice detector computed tomography (MSCT) allows visualisation of human coronary arteries with diagnostic image quality. In addition to conventional axial slices, virtual coronary angioscopies (VCA) can be reconstructed using MSCT datasets.

Objective: To evaluate the feasibility of reconstructing VCA and to determine the clinical value of this new application in detecting atherosclerotic coronary artery lesions.

Methods: Datasets obtained by contrast enhanced non-invasive coronary angiography using MSCT (Somatom VZ) were analysed from 14 consecutive patients. VCA were simulated in 14 coronary arteries (left anterior descending, $n=7$; right coronary, $n=7$ ). Lesion detection was undertaken on conventional contrast enhanced axial slices, as well as by VCA. Intracoronary ultrasound (ICUS) was used as the gold standard for in vivo plaque detection.

Results: 38 lesions were detected both on ICUS and on axial slices: 14 severe target lesions of $>75 \%$ area stenosis (11 calcified, three non-calcified), and 24 intermediate lesions of $\leqslant 75 \%$ area stenosis (seven calcified, 17 non-calcified). Using VCA, all severe lesions $(n=14)$ and all calcified intermediate plaques $(n=7)$ could clearly be identified. However, non-calcified intermediate lesions $(n=17)$ could not be accurately distinguished from the vessel wall; they were recognised as vessel wall alterations without significant luminal narrowing.

Conclusions: Current MSCT technology allows reconstruction of VCA with good image quality. Despite a more anatomical view of heart and coronary vessels on three dimensional reconstruction, conventional axial slices were found to be superior for detecting coronary lesions. Thus further technical innovations are required before VCA can become a useful technique in clinical cardiology.
$\mathrm{R}$ ecent technical innovations in computed tomography and magnetic resonance imaging have opened the field of virtual diagnostics to clinical medicine. ${ }^{12}$ Significantly improved image quality, with high resolution and new software for three dimensional postprocessing, has made non-invasive examination of the cavities within the human body possible. ${ }^{3}$

Promising results have been reported, especially when examining cavities with large interiors, such as the stomach, ${ }^{4}$ the colon, ${ }^{5-7}$ the urinary bladder, ${ }^{8}$ and the bronchi. ${ }^{9}$ Recently, initial results have been reported on coronary artery fly-through using electron beam computed tomography (EBCT). ${ }^{10}$ One major disadvantage of EBCT is that its availability is restricted to about 150 sites worldwide. ${ }^{11}$

Since 1999, conventional mechanical multislice computed tomography (MSCT) systems-with simultaneous acquisition of four slices, $500 \mathrm{~ms}$ scanner rotation time, and down to 125 ms temporal resolution-have been available for general purpose scanning. ${ }^{12}$ Owing to the increased scan speed, thinner slice widths can be achieved in a given volume. Thus $1 \mathrm{~mm}$ slices and submillimetre image increments provide almost isotropic volume data on the heart within a single breath hold. ${ }^{13}{ }^{14}$ Studies focusing on reliability in the detection of coronary artery disease are promising. ${ }^{15-17}$

Our aims in the present study were first, to evaluate feasibility of reconstructing VCA using the new MSCT technology, and second, to determine the clinical value of this new application in detecting atherosclerotic coronary artery lesions. We used intracoronary ultrasound (ICUS) as the gold standard for in vivo plaque detection.

\section{METHODS}

\section{Patients and study protocol}

The study population consisted of 14 consecutive patients with chronic stable angina caused by a severe lesion (>75\% area stenosis) in the proximal vessel segments of the left anterior descending coronary artery $(\mathrm{n}=7)$ or the right coronary artery $(n=7)$. These patients had been assigned on clinical grounds to ICUS guided percutaneous transluminal coronary angioplasty (PTCA).

The study protocol was approved by the local ethics committee, and all patients gave informed consent before their inclusion in the study. Multislice computed tomography of the heart was performed within 24 hours of the intervention. Immediately before PTCA, ICUS was done to analyse the vessel morphology proximal to and at the target lesion. To ensure that the same plaque was assessed by the different techniques and to allow correlations to be made, specific landmarks were used-for example, the origin of side branches and the distance to the target lesion.

\section{Non-invasive coronary angiography and virtual coronary angioscopy}

For MSCT, we used a Somatom Volume Zoom scanner (Siemens, Forchheim, Germany), with a temporal resolution of down to $125 \mathrm{~ms}$ and ECG gated heart phase selective image

Abbreviations: EBCT; electron beam computed tomography; ICUS, intracoronary ultrasound; MSCT, multislice detector computed tomography; PTCA, percutaneous transluminal angioplasty; VCA, virtual coronary angioscopy 


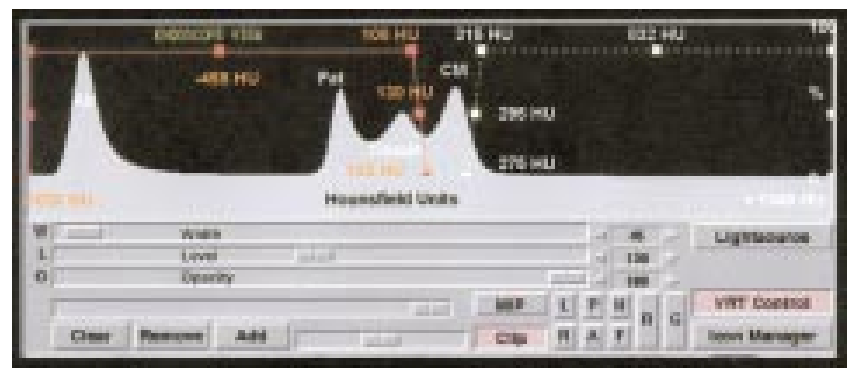

Figure 1 Volume rendering controls with a histogram showing voxel density values. The $x$ axis shows the voxel values, the $y$ axis the frequency distribution. The first peak represents air, the second fat, the third tissue, and the fourth contrast medium (CM). The orange graph represents the range used for visualising vessel, the white graph represents the range used for visualising calcification.

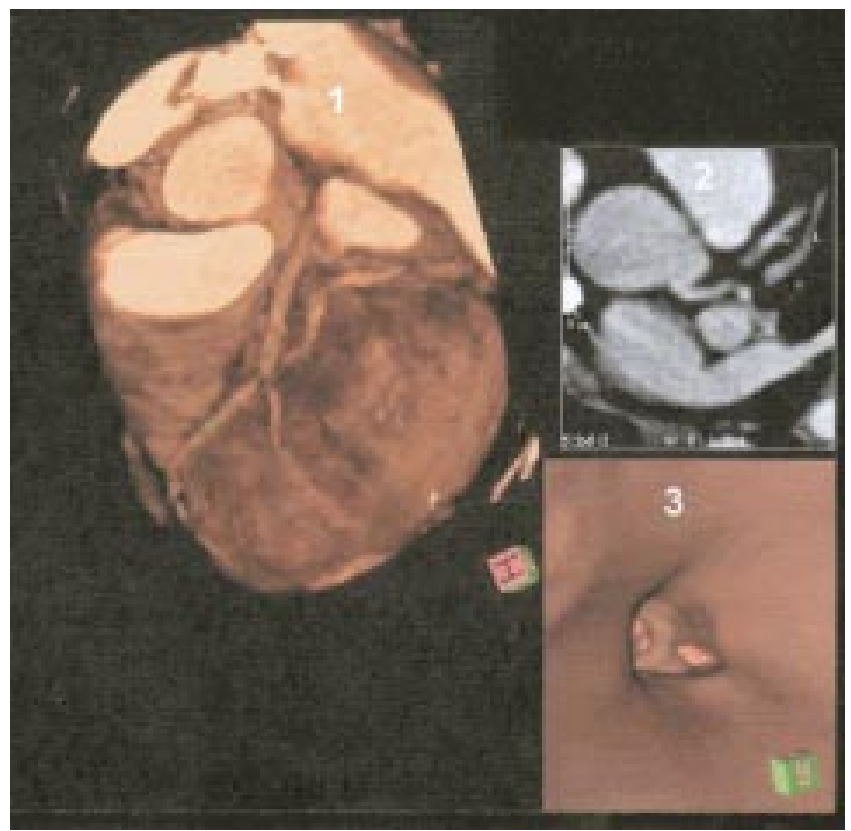

Figure 2 Simultaneous display of three dimensional volume rendering image, axial slice image, and virtual coronary angioscopy. Panel 1: three dimensional volume rendering images showing left anterior descending coronary artery (LAD) with severe stenosis. Panel 2: axial slice image. Panel 3: virtual coronary angioscopy, showing the left main stem and the bifurcation of LAD/left circumflex coronary artery.

reconstruction. For the contrast enhanced scan (collimation $1.0 \mathrm{~mm}$, pitch $1.5,120 \mathrm{kV}, 300 \mathrm{~mA}$, rotation time $500 \mathrm{~ms}$ ), $150 \mathrm{ml}$ of contrast agent $(400 \mathrm{mg} / \mathrm{ml})$ were injected through an 18 gauge catheter into an antecubital vein at $4 \mathrm{ml} / \mathrm{s}$, followed by $30 \mathrm{ml}$ of saline. ${ }^{17}$ All scans were performed during a single breath hold ( $\sim 30$ seconds). The raw scan data were then reconstructed using algorithms optimised for ECG gated multislice spiral reconstruction. ${ }^{13}$ The time point for optimised image reconstruction was determined in a test series. In our series, image reconstruction was conducted in the diastolic phase, using retrospective ECG gating with $38-50 \%$ delay after the onset of the R wave for the right coronary artery, and 50\% delay for the left coronary artery. Between 150 and 200 axial image slices were reconstructed with a $0.8 \mathrm{~mm}$ slice increment and a matrix size of $512 \times 512$, covering the whole volume of the heart with an almost isotropic spatial resolution $(0.6 \times 0.6 \times 0.9 \mathrm{~mm})$. The field of view for image reconstruction was adapted to the heart volume (range $150-200 \mathrm{~mm}$ ), yielding pixel sizes ranging from $0.29 \times 0.29 \mathrm{~mm}$ to $0.39 \times 0.39 \mathrm{~mm}$. The reconstructed image data were transferred to a computer workstation for postprocessing (3DVirtuoso, Siemens, Forchheim, Germany). When performing
Table 1 Patient characteristics and cardiovascular risk factors

\begin{tabular}{ll}
\hline Characteristic & \\
\hline Age (years) (mean (SD); range) & $59(10) ; 41$ to 71 \\
Male sex & $79 \%$ \\
Smoking & $64 \%$ \\
Diabetes mellitus & $21 \%$ \\
Hyperlipidaemia & $71 \%$ \\
Hypertension & $64 \%$ \\
Body mass index $\left(\mathrm{kg} / \mathrm{m}^{2}\right)$ (mean (SD)) & $27(3)$ \\
Family history & $40 \%$ \\
Myocardial infarction & $43 \%$ \\
& \\
Exertional angina pectoris pre-PTCA (CCS classification $\left.{ }^{20}\right)$ \\
I & $64 \%$ \\
II & $29 \%$ \\
III & $7 \%$ \\
IV & 0 \\
\hline
\end{tabular}

CCS, Canadian Cardiovascular Society; PTCA, percutaneous transluminal coronary angioplasty.

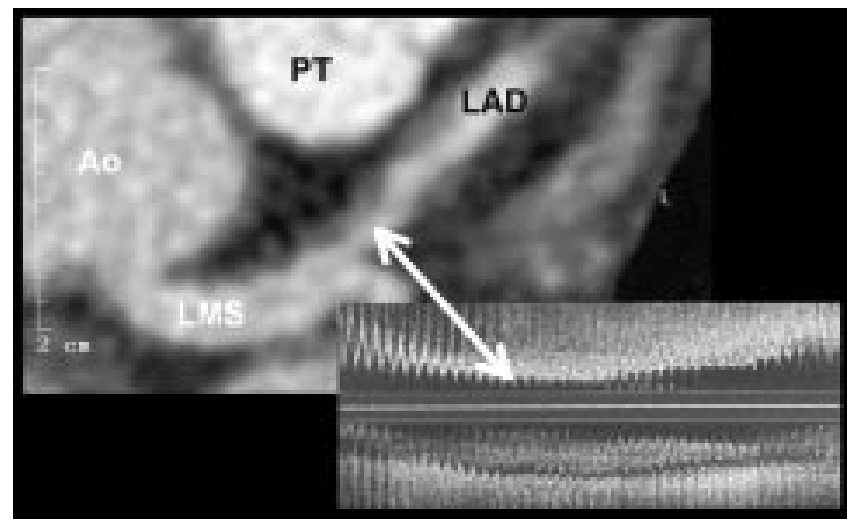

Figure 3 Visualisation of a non-calcified atherosclerotic coronary lesion on conventional contrast enhanced axial slices. Left: contrast enhanced conventional axial slice visualising two non-calcified plaques. Right: corresponding intracoronary ultrasound image (longitudinal scan). Ao, aorta; LAD, left anterior descending coronary artery; LMS, left main stem; PT, pulmonary trunk.

three dimensional volume rendering, the image data are transferred into three dimensional voxels, each of which contains a certain density value, expressed by hounsfield units (HU). Voxels with different HU can be visualised by the use of different colours. Standard 3D-Virtuoso software contains an integrated mode, dedicated to creating volume rendered endoscopic views. To make the vessel lumen visible, contrast medium enhanced blood within the coronary arteries must be hollowed out by excluding the voxels within the lumen (level $\sim 100-200 \mathrm{HU}$ ) from the endoscopic image. The vessel wall is well defined, as it has a significantly lower density than the contrast enhanced lumen (level $\sim 80-100 \mathrm{HU}$ ). Calcifications can be visualised when they are denser than the contrast medium (level $>250 \mathrm{HU}$, fig 1 ). The simultaneous display of three dimensional volume rendered models, multiplanar reformatted (MPR) or axial slice images, and endoscopic views allows accurate interactive navigation of the viewpoint (fig 2). One starts by positioning the navigator at the region of interest; automatic path definition then allows one to find the accurate way from one target point to another, creating the impression of flying through the vessel lumen of the coronary arteries.

\section{Intracoronary ultrasound}

The transfemoral Judkins technique was used in all interventions. Selective angiography was done in multiple views. After passage of the guidewire across the target lesion, ICUS was 


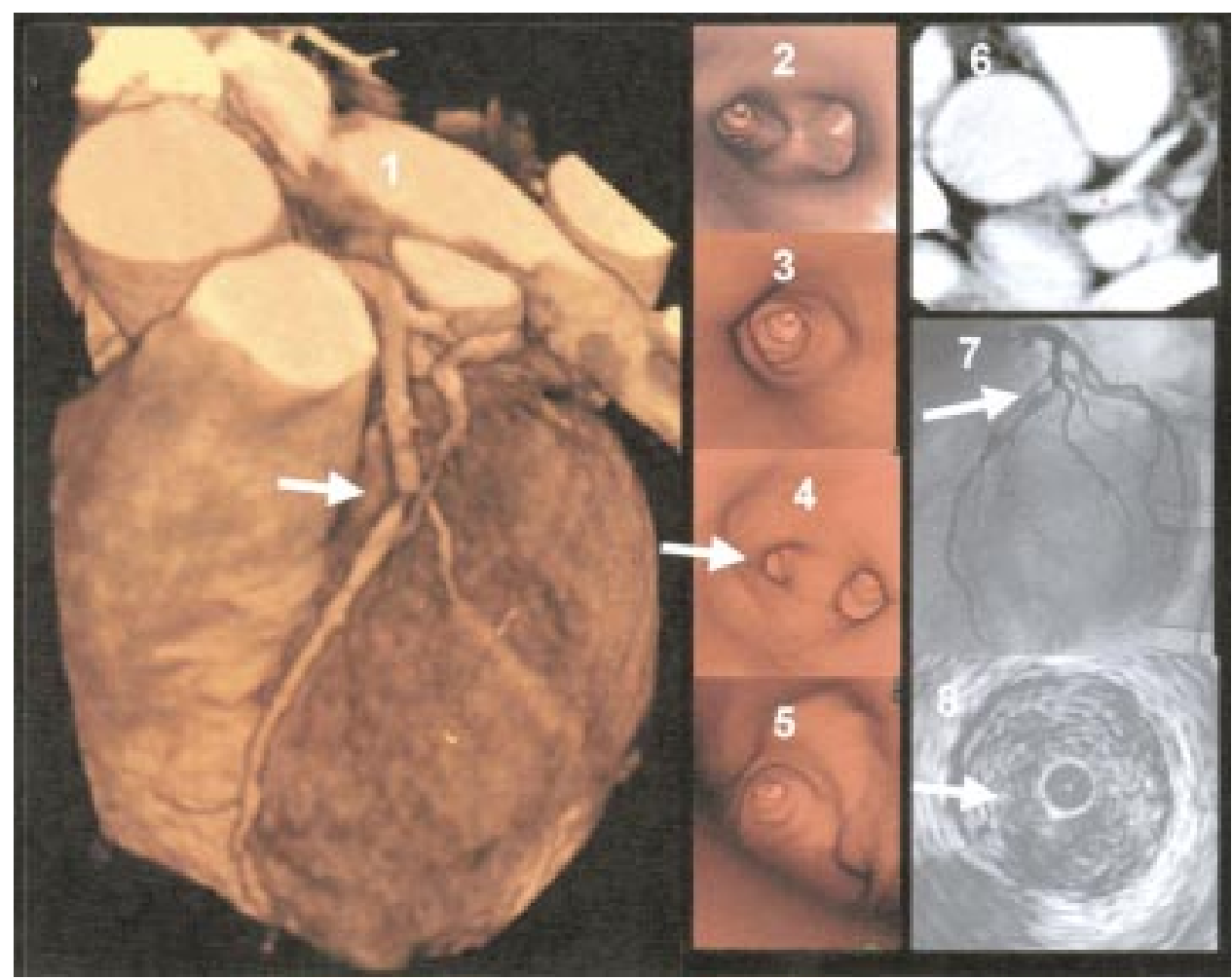

Figure 4 Example of an image from a patient with severe stenosis in the left anterior descending coronary artery. Panel 1: three dimensional volume rendering image, showing a severe lesion in the left anterior descending coronary artery (LAD, marked with an arrow). Panel 2: endoscopic view into the left main stem with visualisation of the $L A D /$ left circumflex coronary artery bifurcation. Panel 3: proximal segment of LAD with vessel wall alterations but non-significant luminal narrowing. Panel 4: view at the severe lesion (on the left) and origin of the diagonal branch (on the right). Panel 5: view from inside the severe lesion into the vessel periphery of the LAD. Panel 6: axial slice image with navigator located in the left main stem. Panel 7: corresponding coronary angiography image, showing the severe LAD lesion. Panel 8: intracoronary ultrasound image within the severe non-calcified lesion.

conducted under fluoroscopic guidance (UltraCross $3.2 \mathrm{~F}$, $30 \mathrm{MHz}$ coronary imaging catheter, SCIMED, Boston Scientific Corporation, San Jose, California, USA). Continuous ultrasound images were received by motorised pullback of the catheter $(0.5 \mathrm{~mm} / \mathrm{s})$ from preferably $10 \mathrm{~mm}$ distal to the target lesion into the guiding catheter. The images were immediately digitalised using echoPlaque software (Indec Systems Inc, Mountain View, California, USA).

\section{Lesion detection}

ICUS was used as an in vivo gold standard for lesion detection within the coronary arteries. ${ }^{18}{ }^{19}$ The analyses were performed in two steps. In step one, plaque detection was performed off line using the ICUS pullback images. Stenosis severity was classified according to per cent area stenosis on axial slices using echoPlaque Software (Indec Systems). In step two, all lesions detected on ICUS were looked for on conventional contrast enhanced axial slices and VCA. Stenosis severity was graded by the observers semiquantitatively, as at present no software for quantitative analyses is available. Lesion detection was performed by two independent observers who were blinded to each other's findings. Doubtful cases were reviewed until consensus could be obtained.

\section{RESULTS}

All 14 MSCT scans and three dimensional datasets provided diagnostic image quality for reconstruction of conventional axial slices and virtual coronary angioscopies and for plaque detection. No patient was excluded from analysis. The patients had a mean (SD) heart rate of 67 (14) beats/min. The clinical characteristics of the patients are summarised in table 1 . To ensure that the identical plaque was assessed by ICUS, by conventional contrast enhanced axial slices, and by VCA, landmarks such as side branches were used for orientation. Bifurcations and side branches could be clearly demonstrated by VCA.

\section{ICUS and conventional axial slices}

We detected 38 lesions on both ICUS and conventional contrast enhanced axial slices (fig 3): 14 severe lesions with $>75 \%$ area stenosis ( 11 calcified, three non-calcified), and 24 intermediate lesions of $\leqslant 75 \%$ area stenosis (seven calcified, 17 non-calcified). The distribution of the segments involved was as follows: segment $1, \mathrm{n}=8$; segment $2, \mathrm{n}=9$; segment $3, \mathrm{n}=2$; segment $5, \mathrm{n}=1$; segment $6, \mathrm{n}=12$; segment 7 , $\mathrm{n}=6$ ).

\section{Virtual coronary angioscopy}

All severe lesions $(n=14)$ could be visualised by VCA, as the lumen diameters within these lesions were significantly narrowed compared with the reference segments (fig 4). Calcifications were clearly detected by VCA in 18 lesions (severe lesions, $\mathrm{n}=1 \mathrm{l}$; intermediate lesions, $\mathrm{n}=7$ ) and could easily be differentiated from the vessel wall. All calcified intermediate lesions were identified $(\mathrm{n}=7$, figs 5,6 , and 7$)$. As indicated in fig 5, information on the morphology of calcified
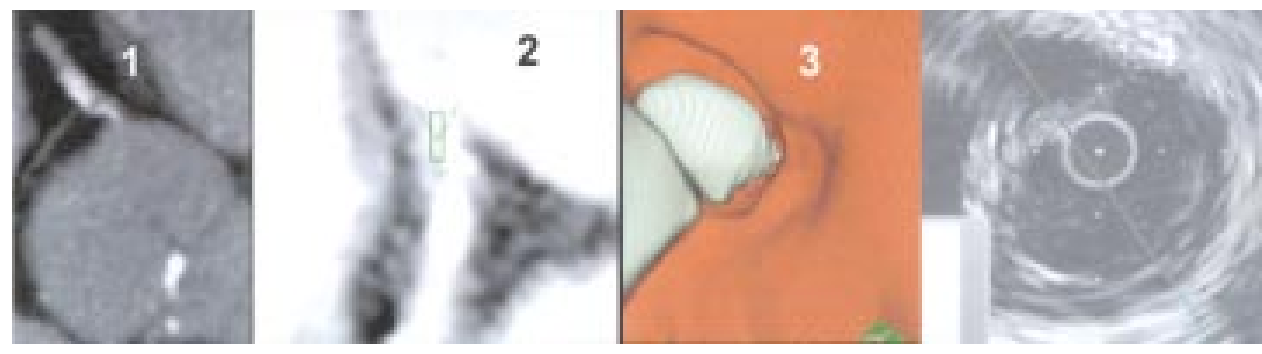

Figure 5 Severe calcifications in the proximal segment in the right coronary artery (RCA). Panel 1: axial view of the proximal segments of the RCA. Panel 2: multiplanar reformatted (MPR) view with navigator, segmented calcification. Panel 3: virtual coronary angioscopy of the corresponding segment with visualisation of the segmentation. Panel 4: corresponding intracoronary ultrasound image with calcification but without severe stenosis. 


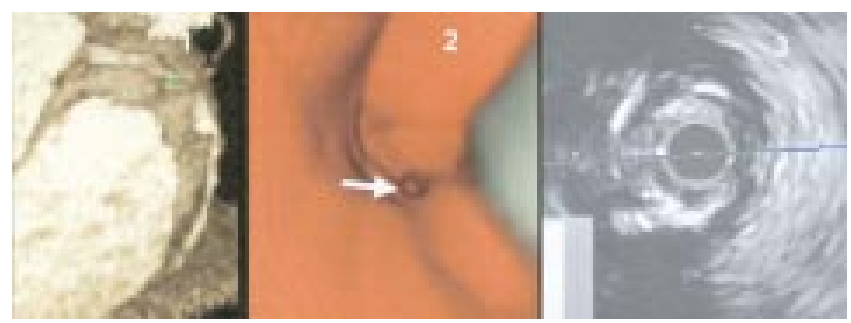

Figure 6 Severe lesion in segment 2 of the right coronary artery. Panel 1: three dimensional volume rendering image of the right coronary artery with severe lesion and calcifications - the navigator is positioned in front of the lesion. Panel 2: virtual coronary angioscopy of the corresponding segment, visualising the severe lesion with calcification. Panel 3: intracoronary ultrasound image of the corresponding segment, visualising the severe stenosis and calcifications.

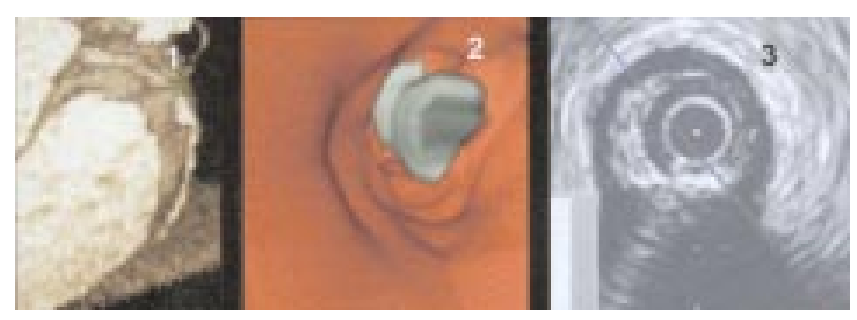

Figure 7 Example of an image from a patient with severe calcifications and a severe stenosis in the right coronary artery. Panel 1: three dimensional volume rendering image of the right coronary artery with high grade stenosis and calcifications-the navigator is positioned immediately beyond the stenosis. Panel 2 : virtual coronary angioscopy view into the distal reference segment with "free floating" calcification in front of the lesion. Panel 3: intracoronary ultrasound image of the corresponding segment, visualising a calcified lesion.

lesions could also be obtained, as segmentation of the plaque could be visualised. Three dimensional reconstruction yielded the impression of "free floating" calcifications within the vessel lumen, preventing the precise determination of plaque position (fig 7$)$. Non-calcified intermediate lesions $(n=17)$ could not be accurately distinguished from the vessel wall, but were recognised as vessel wall alterations without significant lumen narrowing (fig 4).

\section{DISCUSSION}

The new findings of this study are as follows. Firstly, virtual coronary angioscopy can reliably be applied to the MSCT datasets achieved by contrast enhanced non-invasive coronary angiography; current three dimensional postprocessing software allows the reconstruction of virtual coronary angioscopies with good image quality. Secondly, conventional axial slices were found to be superior to VCA for lesion detection, as noncalcified, non-significant lesions can be clearly differentiated from the vessel wall with the former technique (fig 3). ${ }^{21}$

\section{Plaque detection by virtual coronary angioscopy}

Virtual coronary angioscopy was found to be accurate at detecting complex lesions with an irregular surface and calcification, because of the high density of the calcium deposits within the lumen. By contrast, lesions with a smooth surface were poorly visualised by VCA, because non-calcified plaques have a density close to that used to visualise the vessel wall $(<130 \mathrm{HU})$. At present, therefore, non-calcified plaques cannot be accurately differentiated from the vessel wall, which prevents the detection of many mild and intermediate lesions. Non-calcified severe lesions could, however, be identified because they cause significant lumen narrowing (fig 4). Quantitative measurements from VCA - which might be useful for determining stenosis severity or plaque volume-are currently limited by the lack of analysis software. Furthermore, reconstruction of VCA for non-invasive plaque detection is rather time consuming as it takes approximately 60 minutes to reach a diagnosis.

Our data suggest that conventional contrast enhanced axial slices, which are already available 3-5 minutes after the end of data acquisition, are superior to VCA for lesion detection, particularly since non-calcified coronary lesions can be visualised with good diagnostic accuracy. ${ }^{22}{ }^{23}$ Thus a more accurate diagnosis of coronary artery disease is likely to be achieved using conventional contrast enhanced axial slices than VCA. Because of their time consuming nature, VCA should be restricted to doubtful cases where additional three dimensional reconstructions might be helpful-for example, in the evaluation of bifurcation lesions or bypass anastomoses. ${ }^{10}$ However, no data are available on this as yet, and further studies are required.

One major limitation of the application of MSCT in the diagnosis of coronary artery disease is the relatively high radiation dose $\left(\sim 3.9-5.8 \mathrm{mSv}^{15}\right)$ compared with conventional coronary angiography $\left(\sim 3 \mathrm{mSv}^{24}\right)$. As radiation is used at present throughout the heart cycle, additional scan protocols are needed to allow scanning to be done in selected time frames-for example, during diastole-in order to reduce the radiation dose.

As indicated by figs 4-7, image quality may vary in different arteries. The left circumflex coronary artery and the right coronary artery show more rapid diastolic motion, influencing image quality. Thus the reconstruction of these vessels is done during early diastole, while reconstruction of the left anterior descending coronary artery is usually done in mid-diastole. ${ }^{17}$ Despite these limitations, VCA can be applied to right coronary artery datasets with adequate image quality.

\section{Comparison with coronary artery fly-through using electron beam tomography}

Recently, van Ooijen and colleagues presented initial results on coronary artery fly-through imaging using EBCT. In that study, the coronary artery fly-through could only be reconstructed in 10 of 39 cases owing to insufficient image quality in the remaining datasets. ${ }^{24}$ In our series using MSCT, acceptable VCA could be reconstructed in all cases. The significant improvement in image quality of conventional mechanical spiral computed tomography scanners is mainly a result of the recent introduction of multislice technology: MSCT scanners with a $500 \mathrm{~ms}$ rotation time provide a high spatial resolution owing to thin collimated slice widths $(1.0 \mathrm{~mm})$, overlapping image increments, and low image noise..$^{12} 15{ }^{1621}$ Also, temporal resolution is significantly improved (down to $125 \mathrm{~ms}$ ). Dedicated algorithms for retrospective ECG gated reconstruction of raw data allow drastically reduced motion and breathing artefacts, ${ }^{13}$ whereas EBCT technology is restricted to prospective triggering. ${ }^{10}$

\section{Study limitations}

All patients were in sinus rhythm, and proximal segments of the left anterior descending coronary artery and the right coronary artery were studied exclusively. Thus further studies are needed in patients with arrhythmias, and in the left circumflex artery and more distal vessel segments. Furthermore, lesion detection was not undertaken in a double blind manner. However, as it was the aim of the study to evaluate plaque visualisation and not diagnostic accuracy, our principal conclusions remain unaffected. As stated above, the currently high radiation dose must be recognised as a major limitation of non-invasive coronary angiography using MSCT.

\section{Conclusions}

The new MSCT technology provides the option for reconstructions of virtual coronary angioscopies. Despite promising initial results, the clinical application of this new technique was 
found to be limited. VCA is a method of compressing a huge amount of image data into a form of presentation that can be viewed in a short time. Further technical innovations with even thinner slice widths and improved postprocessing software are required to allow a more precise angioscopic view of the coronary vessel wall, before virtual coronary angioscopy can become a useful application in clinical cardiology.

\section{Authors' affiliations}

S Schroeder, A Kuettner, C Herdeg, Department of Internal Medicine, Division of Cardiology Eberhard-Karls-University, Tuebingen, Germany

A F Kopp, C D Claussen, Department of Radiology, Division of Diagnostic Radiology, Eberhard-Karls-University

B Ohnesorge, H Loke-Gie, Siemens AG, Medical Engineering,

Computed Tomography, Forchheim, Germany

A Baumbach, K R Karsch, Department of Cardiology, Bristol Heart Institute, University of Bristol, United Kingdom

\section{REFERENCES}

1 Ackerman JD. Medicine meets virtual reality 2000. MD Comput 2000;17:13-17.

2 Fletcher JG, Luboldt W. CT colonography and MR colonography: current status, research directions and comparison. Eur Radiol 2000;10:786-801.

3 Potchen EJ. Prospects for progress in diagnostic imaging. J Intern Med 2000;247:411-24.

4 Ott DJ. Virtual gastroscopy: a new look at the stomach. Am J Gastroenterol 2000;95: 1084-5

5 Chaoui AS, Blake MA, Barish MA, et al. Virtual colonoscopy and colorectal cancer screening. Abdom Imaging 2000;25:361-7.

6 Pescatore P, Glucker T, Delarive J, et al. Diagnostic accuracy and interobserver agreement of CT colonography (virtual colonoscopy). Gut 2000;47: 126-30.

7 Schoenfelder D, Debatin JF. Virtual reality in MR colonography Radiologe 2000;40:283-9.

8 Schreyer AG, Fielding JR, Warfield SK, et al. Virtual CT cystoscopy: color mapping of bladder wall thickness. Invest Radiol 2000;35:331-4

9 Rapp-Bernhardt U, Welte T, Doehring W, et al. Diagnostic potential of virtual bronchoscopy: advantages in comparison with axial CT slices, MPR and mIP? Eur Radiol 2000;10:981-8.
10 van Ooijen PM, Oudkerk M, van Geuns R, et al. Coronary artery fly-through using electron beam computed tomography. Circulation 2000;102:E6-10.

11 Sechtem U. Electron beam computed tomography: on its way into mainstream cardiology? Eur Heart J 2000;21:87-91

12 Klingenbeck-Regn K, Schaller S, Flohr T, et al. Subsecond multislice computed tomography: basics and applications. Eur J Radiol 1999:31:110-24.

13 Ohnesorge B, Flohr T, Becker CR, et al. Cardiac imaging by means of electrocardiographically gated multisection spiral $\mathrm{CT}$ : initial experience. Radiology 2000;217:564-71.

14 Ohnesorge B, Flohr T, Becker C, et al. Cardiac imaging with rapid, retrospective ECG synchronized multilevel spiral CT. Radiologe 2000;40:111-17.

15 Achenbach S, Ulzheimer S, Baum U, et al. Noninvasive coronary angiography by retrospectively ECG-gated multislice spiral CT. Circulation 2000; 102:2823-8.

16 Knez A, Becker C, Ohnesorge B, et al. Noninvasive detection of coronary artery stenosis by multislice helical computed tomography. Circulation 2000;101:E221-2.

17 Kopp AF, Ohnesorge B, Flohr T, et al. Cardiac multidetector-row CT: first clinical results of retrospectively ECG-gated spiral with optimized temporal and spatial resolution. Fortschr Rontgenstr 2000;172:429-35.

18 Schroeder S, Baumbach A, Mahrholdt H, et al. The impact of untreated coronary dissections on acute and long-term outcome after intravascular ultrasound guided PTCA. Eur Heart J 2000;21:137-45.

19 Yamagishi M, Terashima M, Awano K, et al. Morphology of vulnerable coronary plaque: insights from follow-up of patients examined by intravascular ultrasound before an acute coronary syndrome. J Am Coll Cardiol 2000;35:106-11.

20 Campeau L. Grading of angina pectoris [letter]. Circulation 1976:54:522-3.

21 Becker CR, Knez A, Ohnesorge B, et al. Imaging of noncalcified coronary plaques using helical CT with retrospective ECG gating. Am J Roentgenol 2000;175:423-4.

22 Schroeder S, Kopp AF, Baumbach A, et al. Noninvasive detection and evaluation of atherosclerotic coronary plaques with multislice computed tomography. J Am Coll Cardiol 2001;37:1430-5.

23 Schroeder S, Kopp AF, Baumbach A, et al. Non-invasive detection of coronary lesions by multi-slice computed tomography: results of the new-age pilot trial. Cathet Cardiovasc Intervent 2001;53:352-8.

24 Becker C, Schatzl M, Feist $\mathrm{H}$, et al. Assessment of the effective dose for routine protocols in conventional CT, electron beam CT and coronary angiography [in German]. Fortschr Rontgenstr 1999;170:99-104.

\section{IMAGES IN CARDIOLOGY}

\section{Incorrect administration of buccal glyceryl trinitrate}

A

66 year old man with documented coronary artery disease was brought by ambulance to the emergency department with severe anginal chest pain and pulmonary oedema. Two $2 \mathrm{mg}$ buccal glyceryl trinitrate (GTN) (Buccal Suscard) tablets had been inserted under the top lip by paramedics as per their protocol. Symptoms did not resolve and shortly after arrival in hospital he suffered cardiac arrest from which attempts at resuscitation were unsuccessful. On removing the dentures before intubation it was noticed that the tablets has become adherent to the outside of the upper denture, thus reducing the available surface area of mucosa through which this medication can be absorbed. The manufacturer reports that the primary site for absorption of buccal GTN (Buccal Suscard) is through the gum mucosa, and should be administered under any denture plate that is fitted in order to maximise therapeutic effect. Incorrect administration of this medication is commonplace and may have adversely affected the outcome of this case.

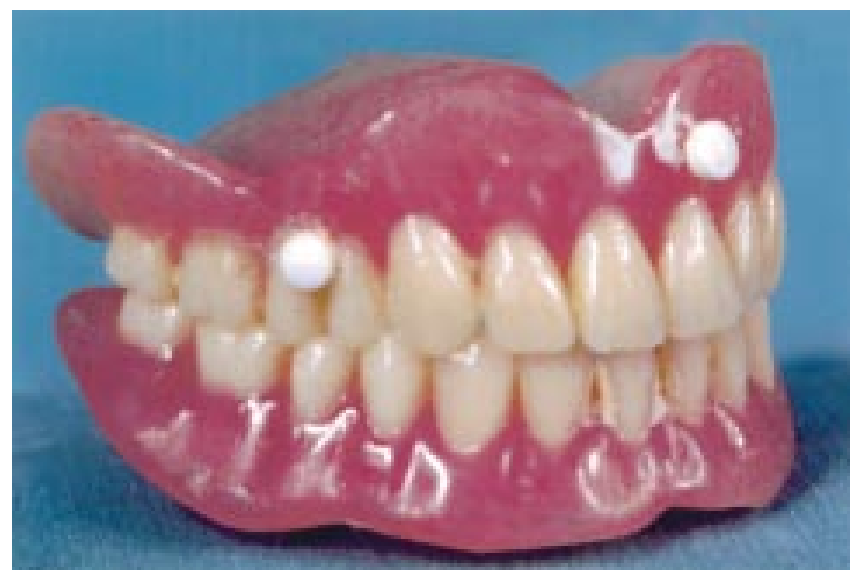

A Watson

L Michaels

AlanRWatson@aol.com 